\title{
Adaptação ao Cancro da Mama: Validação da Versão Portuguesa da Psychological Adaption Scale
}

\author{
Adaptation to Breast Cancer: Validation of the Portuguese Version of Psychological \\ Adaptation Scale
}

\author{
Vera Neto $^{1}$, Catherine Jönsson ${ }^{2}$, Sónia Castro $^{3}$, Eunice R. Silva ${ }^{4}$ e Leonor Lencastre ${ }^{5}$
}

\begin{abstract}
Resumo
A doença oncológica pode implicar consequências físicas, psicológicas e sociais que poderão causar desestruturações na vida dos doentes. A adaptação à doença é um desafio que pode passar pela mudança de comportamentos, atitudes e pensamentos, abrangendo diferentes domínios. Detetar e identificar padrões de adaptação disfuncionais no decurso da doença oncológica é fundamental para a prevenção e intervenção na inadaptação à doença. O objetivo da presente investigação foca-se na validação da versão portuguesa da Psychological Adaptation Scale (PAS), de Biesecker et al. (2013), que visa avaliar a adaptação à doença em domínios específicos. Recorreu-se a uma amostra por conveniência de 98 mulheres com cancro da mama. Obteve-se uma nova estrutura fatorial, com 3 subescalas dotadas de validade externa e de valores elevados de fiabilidade. A PAS surge como um instrumento válido na caracterização da adaptação à doença oncológica, assim como na identificação de domínios específicos da adaptação a serem alvo de intervenção.
\end{abstract}

Palavras-chave: adaptação psicológica, cancro, qualidade de vida, bem-estar, validação instrumentos

\begin{abstract}
Cancer disease may impose physical, psychological and social barriers, which can cause disruptions in the lives of cancer patients. Adapting to the disease is a challenge that may involve changing behaviors, attitudes and thoughts, covering different domains. Therefore, detecting and identifying dysfunctional adaptation patterns in the course of the cancer disease is essential for the prevention and intervention in the case of inadaptation to the disease. The objective of the present investigation focuses on the validation of the Psychological Adaptation Scale (PAS), by Biesecker et al. (2013), which aims to assess adaptation to the disease in specific domains. A convenience sample of 98 women with breast cancer was used. A new factorial structure of 3 subscales was obtained, with external validity and high reliability values. Thus, the PAS appears as a valid instrument for the characterization of adaptation to cancer disease and also for the identification of specific domains of adaptation that may need intervention.
\end{abstract}

Keywords: psychological adaptation, cancer disease, quality of life, well-being, instrument validation

Financiamento: Este estudo foi financiado pelo Centro de Psicologia da Universidade do Porto, Fundação para a Ciência e Tecnologia Portugal (FCT UIDB/00050/2020)

\footnotetext{
${ }^{1}$ Mestre em Psicologia Clínica e da Saúde pela FPCEUP - Faculdade de Psicologia e de Ciências da Educação da Universidade do Porto. Rua Alfredo Allen, 4200-135, Porto, Portugal. Tel.: +351913692878. E-mail: vera_neto_24@ hotmail.com (Autor de correspondencia)

${ }^{2}$ Doutorada em Psicologia pela FPCEUP - Faculdade de Psicologia e de Ciências da Educação da Universidade do Porto. Rua Alfredo Allen, 4200-135, Porto, Portugal.

${ }^{3}$ Mestre em Psicologia Clínica e da Saúde pela FPCEUP, Psicóloga Clínica e da Saúde no IPO - Instituto Português de Oncologia do Porto FG, EPE.

${ }^{4}$ Doutorada em Psicologia pela FPCEUP, Coordenadora do Serviço de Psicologia no IPO do Porto FG, EPE.

${ }^{5}$ Doutorada em Psicologia pela FPCEUP, Investigadora do Centro de Psicologia da Universidade do Porto, Prof ${ }^{a}$ Associada na FPCEUP, Rua Alfredo Allen, 4200-135, Porto, Portugal. E-mail: leonor@fpce.up.pt (Autor de correspondencia)
}

Revista Iberoamericana de Diagnóstico y Evaluación - e Avaliação Psicológica. RIDEP · No60 • Vol.3 · 55-69 2021

ISSN: 1135-3848 print /2183-6051online 


\section{Introdução}

A doença oncológica é um problema de saúde pública emergente na sociedade atual. Os avanços da ciência e da tecnologia têm permitido uma atuação mais eficaz junto destes doentes, levando por consequência a consideráveis aumentos das taxas de sobrevivência (Brazão, 2020). Apesar da doença oncológica ser uma doença física, esta pode implicar sérias consequências psicológicas que poderão vulnerabilizar os doentes (Albert et al., 2016; Dumalaon-Canaria et al., 2018; Pinto \& Pais-Ribeiro, 2007).

O cancro da mama apresenta uma elevada incidência nos países desenvolvidos, sendo as doentes com este tipo de cancro frequentemente confrontadas com sequelas graves, quer físicas quer emocionais, que obrigam a reajustamentos profundos nas suas vidas (Antoni et al., 2009; Brandão \& Matos, 2015; Dukes-Holland \& Holahan, 2003; Moreira et al., 2008; Silva et al., 2009). As exigências físicas e psicológicas que o cancro da mama impõe a estas mulheres, podem desencadear sofrimento emocional ou distress, que se caracteriza por sentimentos de ansiedade, depressão, medo, desespero e tristeza (Holland, 2010; Riba, et al., 2019; National Comprehensive Cancer Network, [NCCN] 2021). Cada diagnóstico é único e vivido de forma pessoal, podendo provocar as reações mais diversas (Brandão \& Matos, 2015; Larsen, 2014). Há pacientes que no decorrer do processo da doença vão conseguindo desenvolver estratégias de coping mais ou menos adequadas às suas necessidades, transformando as reações iniciais menos adaptativas em reações mais adaptativas, e conseguindo como tal uma melhor adaptação psicológica (AP) à doença (Brandão et al., 2017; Dukes-Holland \& Holahan, 2003). Há no entanto também, um grande grupo de mulheres que não consegue desenvolver estratégias de coping adequadas, prevalecendo sentimentos como a raiva, a culpa, a vergonha, e apresentando frequentemente dificuldades de concentração, insónia, insatisfação com a imagem corporal, ansiedade face à alteração dos papéis sociais, isolamento social, medo de progressão da doença e depressão (Brandão \& Matos, 2015; DukesHolland \& Holahan, 2003).

A AP à doença surge assim, como um processo dinâmico e multidimensional que inclui aspetos inter e intrapessoais, que variam consoante as características individuais, da doença e do meio, correspondendo à interação de diferentes variáveis (Brandão et al., 2017; Kang et al., 2020; Souza, 2014). Tendo em conta a complexidade do constructo da AP, e com o intuito de o tornar mensurável é fundamental refletir sobre os seus principais componentes.

Biesecker et al. (2013) dedicaram especial atenção à questão da avaliação da AP tendo salientado a importância de dois modelos para ajudar na identificação desses componentes: o Modelo Transacional de Stress e Coping de Lazarus e Folkman (1984) que explica a importância do coping na intensidade emocional que a disrupção de uma doença pode suscitar e a Teoria Cognitiva da Adaptação de Taylor (1983) que enfatiza a importância do meaning making ou seja da atribuição de um significado à crise.

Segundo o Modelo Transacional de Stress e Coping, o processo de adaptação a um evento stressor depende da avaliação que o individuo faz do evento e dos recursos físicos e psicológicos de que dispõe para o enfrentar. Estes recursos possibilitam a construção de estratégias para lidar com o stress que o processo adaptativo à doença pode suscitar. Estas estratégias são denominadas estratégias de coping e surgem como as principais mediadoras do processo adaptativo, constituindo um recurso vital para o enfrentamento da doença. As estratégias de coping que parecem ser mais adequadas à AP, são a aceitação e $\mathrm{o}$ enfrentamento conjugado com o espírito de luta, enquanto a negação e o evitamento tendem a suscitar piores estilos de adaptação assim como suscitar maiores níveis de ansiedade e depressão (Brandão et al., 2017; Friedman et al., 1991; Kang et al., 2020; Torres et al., 2014). Este modelo encara assim a adaptação bem-sucedida à doença como uma forma positiva de responder a uma ameaça à saúde. Assim, a eficácia do coping poderá ser encarada como uma dimensão da AP a ter em conta quando se pretende avaliar a mesma.

A Teoria Cognitiva da Adaptação de Taylor (1983) refere que perante uma adversidade stressora, como é o caso de uma doença, o individuo tenta atribuir significado à crise, de forma a tentar percebê-la e a prevenir o impacto que esta poderá ter na sua vida (Loeffler et al., 
2018). A atribuição de um significado positivo ao evento stressor pode levar o individuo a percecionar um maior autocontrole sobre a sua vida, potenciando por sua vez o seu bem-estar espiritual (BEE). Para além da importância do coping, referida no modelo de Lazarus e Folkman (1984) e da atribuição de significado à crise referido na Teoria Cognitiva da Adaptação de Taylor (1983) este último autor salienta ainda a importância da autoestima no processo de AP, realçando que as grandes crises, geralmente têm fortes implicações na perceção que cada doente tem sobre si próprio afetando consequentemente a sua autoestima. Uma baixa autoestima acaba por afetar outros aspetos relevantes na vida do doente, como é o caso dos seus relacionamentos sociais, levando com frequência ao isolamento social, podendo os familiares mais próximos desempenhar um papel preponderante neste domínio (Ávila et al., 2015; Kang et al., 2020; Ponte \& Pais-Ribeiro, 2016). A integração social aparece assim como outro componente importante a ter em conta quando se pretende caracterizar a AP.

No processo de construção da PAS, e especificamente no que se refere à identificação dos principais componentes da AP, Biesecker, et al. (2013) inspirados no Modelo Transacional de Stress e Coping de Lazarus e Folkman e na Teoria Cognitiva da Adaptação de Taylor, chegam assim à identificação de quatro domínios: o coping eficaz, a autoestima, o BEE e a integração social.

A identificação dos componentes da AP tem grandes vantagens ao possibilitar a despistagem precoce de eventuais padrões de adaptação mais pobres e possibilitando intervenções mais específicas no desenvolvimento das competências necessárias para lidar com a doença oncológica (Lang-Rollin \& Berberich, 2018).

Ao longo do tempo a AP à doença tem sido avaliada através de instrumentos que na verdade o que avaliam são constructos relacionados com a mesma, como é o caso da qualidade de vida (QdV) e do bem-estar (BE), estando a AP frequentemente incluída e operacionalizada nesses construtos (Ávila et al., 2015; Heim et al., 1997). Pensa-se, no entanto, que o conceito de AP deverá ser encarado como um constructo distinto destes, apesar de estabelecer com eles uma relação positiva (Borsa et al., 2012; Cotton et al., 1999).
A presente investigação surge com o objetivo de validar uma versão portuguesa da Psychological Adaptation Scale (PAS) criada originalmente por Biesecker et al. (2013), (ver Anexo 1) para avaliar a AP de pessoas em condição de doença crónica ou de risco, e que integra quatro domínios: Eficácia do Coping, Autoestima, BEE e Integração Social. Este estudo pretende validar a versão portuguesa da PAS numa amostra de doentes oncológicas com cancro da mama.

\section{Método}

\section{Participantes}

O presente estudo é composto por uma amostra por conveniência de 98 mulheres diagnosticadas com cancro da mama. As participantes foram recrutadas no Instituto Português de Oncologia (IPO) do Porto, especificamente na Clínica da Mama. Após serem informadas sobre o objetivo do estudo e ser assegurado o anonimato da participação, as participantes preencheram os questionários e procederam à assinatura do consentimento informado, sendo agradecida no fim a colaboração.

Quanto à caracterização sociodemográfica da amostra, a idade das participantes está compreendida entre os 32 e os 75 anos $(M=53.03$, $D P=9.33)$, a maioria é casada (74.5\%) e tem dois filhos (39.8\%). Relativamente às habilitações literárias, $48 \%$ concluiu o ensino secundário ou ensino superior (licenciatura) e $34.7 \%$ apresenta habilitações literárias iguais ou inferiores ao $2^{\circ}$ ciclo do ensino básico completo. Quanto à situação profissional apenas $28.5 \%$ das mulheres se encontram desempregadas ou reformadas, enquanto as restantes estão no ativo ou de baixa médica.

Quanto à caracterização clínica, o tempo desde a obtenção do diagnóstico e o tempo desde o início dos tratamentos correspondem respetivamente a uma média de 36.71 meses $(D P=45.40)$ e 34.23 meses $(D P=45.40)$, equivalendo a uma média de cerca de 3 anos. Relativamente ao estádio da doença apenas $55.1 \%$ das mulheres referiu ter conhecimento desta informação. Dentro destes $55.1 \%$, o estádio mais prevalente foi o estádio III (22.4\%). Quanto à 
existência ou não de metástases, $89.8 \%$ das mulheres respondeu à questão, e destas $15.3 \%$ referiu a sua presença. Quanto aos tratamentos realizados, $73.5 \%$ foi submetida a cirurgia, $58.1 \%$ realizou ou ainda se encontra a realizar quimioterapia, e $55.1 \%$ realizou ou encontra-se ainda a realizar radioterapia.

Finalmente, $22.4 \%$ das mulheres manifestou ter recebido acompanhamento psicológico durante o período da doença oncológica.

\section{Instrumentos}

Neste estudo recorreu-se a 6 instrumentos de avaliação sendo eles: um questionário sociodemográfico e clínico construído pelos autores; a versão portuguesa de Vaz Serra et al. (2006) do World Health Organization Quality of Life - Bref (WHOQOL-Bref) para avaliar a QdV; a versão portuguesa de Cella et al. (1993) do Functional Assessment of Cancer Therapy General (FACT-G) para avaliar a QdV relacionada com a saúde especificada em quatro domínios de BE: BE Físico, BE Social, BE Emocional e BE Funcional; a versão portuguesa de Pereira e Santos (2011) do Functional Assessment of Chronic Illness Therapy - Spiritual Well-Being (FACIT-Sp) para avaliar o BEE; a versão portuguesa de Pais-Ribeiro, Honrado e Leal (2004) da Depression Anxiety Stress Scales (DASS); e finalmente, para a avaliação da AP recorreu-se à Psychological Adaptation Scale (PAS) (ver Anexo 1) instrumento originalmente construído por Biesecker et al. (2013) e traduzido e adaptado para português por Jönsson (2018). No presente estudo, foi usada a versão traduzida por Jönsson (2018) com os 20 itens da versão original.

A PAS é constituída por 4 subescalas ("Eficácia do Coping", "Autoestima", "Bem-estar espiritual" e "Integração Social"). Cada subescala apresenta 5 itens, perfazendo um total de 20 itens. Os itens da PAS seguem uma escala de tipo Likert de 1 "De forma nenhuma" a 5 "Muitíssimo", em que resultados mais elevados correspondem a níveis mais elevados de AP. A soma dos itens de cada subescala da PAS traduz-se num resultado que pode variar entre 5 a 25 pontos. A escala total compreende a soma dos valores de cada uma das subescalas, podendo assim assumir valores entre os 20 e 100 pontos.
Quanto às qualidades psicométricas do instrumento original, aplicado a 6 populações diferentes: adultos com Neurofibromatose Tipo I; homens e rapazes com Síndrome de Klinefelter (XXY); adultos em risco de doença de Huntington; pais de crianças com Perturbação do Espetro do Autismo; pais de crianças com Síndrome de Down; e pais de crianças com Síndrome de Rett, foi verificado que a escala era detentora de uma boa consistência interna $(\alpha=.96)$ (Biesecker et al., 2013). Os autores verificaram que todos os itens se correlacionavam com os seus domínios e que avaliam efetivamente o seu propósito, concluindo que se tratava de um instrumento válido e fiável. (Biesecker et al., 2013).

Em 2018, Jönsson realizou um primeiro estudo de validação de uma versão portuguesa desta escala. Para a tradução e adaptação do instrumento original, e obtenção de um instrumento equivalente ao original em termos linguísticos e culturais, Jönsson (2018) baseou-se nas orientações propostas pela Comissão Internacional de Testes (Tanzer \& Sim, 1999), e também nos 8 passos para a tradução e adaptação de instrumentos psicológicos de Gudmundsson (2009) (ver Anexo 2).

No estudo de Jönsson (2018), em que foi utilizada uma amostra de 150 pessoas que relataram acontecimentos de vida stressantes, não sendo especificamente portadores de doença, a escala demonstrou ter uma estrutura diferente da do estudo original, apresentando 3 subescalas, nomeadamente a subescala da "Autoestima e do Bem-estar espiritual", com 10 itens, a subescala "Integração Social" com 5 itens, e a subescala "Eficácia do Coping" com 4 itens, perfazendo um total de 19 itens, tendo o item 5 sido eliminado por não ser congruente com a estrutura fatorial encontrada. Esta estrutura fatorial apresentou qualidades psicométricas válidas e fiáveis, e as 3 subescalas apresentaram valores de alfa de Cronbach elevados, variando entre .82 e .94 . Para a escala total, foi obtido um valor de alfa de Cronbach de .96 revelando uma boa consistência interna, sendo os resultados obtidos congruentes com os resultados encontrados por Biesecker (Jönsson, 2018). 


\section{Procedimento de análise de dados}

Para o tratamento dos dados recolhidos utilizou-se o programa Statistical Package for the Social Sciences (SPSS) v25 (IBM SPSS, 2017). Procedeu-se a análises descritivas, recorreu-se a análises de diferenças de médias através de testes $t$ de student e da ANOVA. Foram realizadas correlações bivariadas através do recurso ao coeficiente de correlação momento-produto de Pearson. Recorreu-se à Análise de Componentes Principais (ACP) ao alfa de Cronbach, ao teste de Kaiser-Meyer-Olkin (KMO) e ao teste de esfericidade de Bartlett. Importa referir que o valor considerado para o nível de significância foi $p \leq .05$.

\section{Resultados e Discussão}

O primeiro ponto desta secção de apresentação e discussão de resultados será dedicado à validação da PAS, sendo subdividido em 3 pontos: validade interna, fiabilidade $\mathrm{e}$ validade externa. O segundo ponto dedica-se à caracterização da PAS fazendo-se referência às principais medidas descritivas.

\section{Validação da PAS}

Num primeiro ponto será analisada a validade interna através da realização de uma análise fatorial exploratória. Um segundo ponto será dedicado à análise da fiabilidade do instrumento através da análise da consistência interna. Num terceiro ponto analisar-se-á a validade externa através da determinação dos valores das correlações do coeficiente de correlação momento produto de Pearson com outras medidas.

\section{Validade Interna}

Biesecker et al. (2013) no estudo original de validação da PAS chegaram a uma estrutura fatorial de 4 fatores com 5 itens cada, denominados: "Eficácia do Coping", "Autoestima", "Integração Social" e "Bem-Estar Espiritual", tendo obtido valores de consistência interna elevados. Já no estudo de Jönsson de 2018, de validação de uma versão portuguesa dessa mesma escala, a autora chegou a uma estrutura fatorial de 3 componentes: "Eficácia do Coping" com 4 itens, "Autoestima e Bem-Estar Espiritual" composta por 10 itens, que combina os dois fatores originais num só, e "Integração Social" com 5 itens. Nesse estudo a escala total evidenciou um valor de $\alpha$ de Cronbach de .96, igual ao valor encontrado no estudo de Biesecker et al. (2013), e as subescalas apresentaram valores de consistência interna de $\alpha$ de Cronbach entre .82 e 94 .

Antes de proceder à análise de validação da escala, verificou-se a eventual existência de desvios da normalidade mais acentuados, considerando para o efeito, valores de assimetria e da curtose fora do intervalo -2 a 2 (IBM SPSS, 2017). Ao fazer essa análise para os diferentes itens da PAS, verificou-se que todos os valores se encontravam dentro dos valores estipulados. (ver Quadro 1).

Para analisar a validade interna da PAS, e especificamente para a análise da sua estrutura, ou seja, da forma como os itens se relacionavam, recorreu-se a uma análise fatorial exploratória. Recorreu-se ao KMO, para se obter informação sobre a adequação da amostra tendo-se obtido um valor igual a .92, o que nos revelou que a amostra era adequada para a solução fatorial encontrada (Field, 2013). Quanto ao Teste de Esfericidade de Bartlett observaram-se correlações fiáveis, entre os vários itens da escala apresentando um valor estatisticamente significativo $\left(\chi^{2}=1957.58\right.$ (190), $p<.001)$.

A Análise de Componentes Principais (ACP), usando o critério de Kaiser como referência (valores eigen superiores a 1 para a extração dos componentes), revelou 3 componentes. $\mathrm{O}$ primeiro composto por 9 itens, o segundo por $6 \mathrm{e}$ o terceiro por 5 itens, apresentando na sua totalidade uma variância total explicada de $74.65 \%$. O primeiro componente explica $59.54 \%$ da variância (valor eigen=11.91), o segundo explica $9.09 \%$ (valor eigen=1.82), e por fim, o terceiro explica $6.03 \%$ da variância (valor eigen=1.21) (ver Quadro 1).

A rotação Oblimin mostrou depois, os itens com cargas fatoriais mais elevadas em cada um dos componentes (ver Quadro 2). Assim, o primeiro componente revelou saturação nos primeiros 9 itens $(\mathrm{Q} 1$ a $\mathrm{Q} 9)$ com cargas fatoriais superiores a .60. No estudo de Biesecker et al. (2013) os primeiros 5 (Q1 a Q5) pertenciam à subescala da "Eficácia do coping" e os restantes 4 (Q6 a Q9) pertenciam à subescala da "Autoestima" 
Quadro 1. Psychological Adaptation Scale (PAS): Resultados descritivos (N=98)

\begin{tabular}{|c|c|c|c|c|c|}
\hline Dimensões & Itens & $M(D P)$ & $\begin{array}{l}\text { Correlações } \\
\text { Item-Total }(r) \\
\end{array}$ & Assimetria & Curtose \\
\hline \multirow{9}{*}{$\begin{array}{l}\text { Componente } 1 \\
\text { "Eficácia do Coping e Autoestima" } \\
(M=31.95 ; D P=8.79 ; \\
\text { Min-Máx. }=9-45 ; \\
\alpha=.95 \text {; Variância explicada: } 59.54 \%) \text {. }\end{array}$} & Q1 & $3.35(1.11)$ & .84 & -.68 & -.27 \\
\hline & Q2 & $3.44(1.17)$ & .82 & -.84 & -.10 \\
\hline & Q3 & $3.50(1.14)$ & .70 & -.57 & -.48 \\
\hline & Q4 & $3.62(1.08$ & .81 & -.95 & .50 \\
\hline & Q5 & $3.57(1.23)$ & .82 & -.93 & -.14 \\
\hline & Q6 & $3.58(1.18)$ & .78 & -1.04 & .36 \\
\hline & Q7 & $3.31(1.27)$ & .82 & -.57 & -.61 \\
\hline & Q8 & $3.82(1.13)$ & .82 & -1.06 & .61 \\
\hline & Q9 & $3.64(1.12)$ & .84 & -.94 & .45 \\
\hline \multirow{6}{*}{$\begin{array}{l}\text { Componente } 2 \\
\text { "Integração Social" } \\
(M=21.74 ; D P=6.16 ; \\
\text { Min-Máx.=6-30; } \\
\alpha=.92 ; \text { Variância explicada: } 9.09 \%)\end{array}$} & Q10 & $3.22(1.30)$ & .82 & -.60 & -.76 \\
\hline & Q11 & $3.83(1.09)$ & .55 & -.45 & .55 \\
\hline & Q12 & $3.42(1.28)$ & .81 & -.74 & -.56 \\
\hline & Q13 & $3.61(1.17)$ & .81 & -.88 & .09 \\
\hline & Q14 & $3.80(1.22)$ & .85 & -1.14 & .50 \\
\hline & Q15 & $3.87(1.19)$ & .82 & -1.28 & .87 \\
\hline Componente 3 & Q16 & $3.84(1.20)$ & .81 & -1.33 & 1.06 \\
\hline "Bem-Estar Espiritual" & Q17 & $3.76(1.22)$ & .85 & -1.02 & .18 \\
\hline$(M=17.98 ; D P=5.54$ & Q18 & $3.67(1.23)$ & .88 & -1.00 & .19 \\
\hline Min-Máx.= 5-25; & Q19 & $3.26(1.37)$ & .78 & -.53 & -.95 \\
\hline$\alpha=.92 ;$ Variância explicada: $6.03 \%$ ) & Q20 & $3.46(1.35)$ & .67 & -.61 & -.78 \\
\hline $\begin{array}{l}\text { PAS Total } \\
\text { (Variância total explicada: } 74.65 \% \text { ) }\end{array}$ & - & 71,48 (18.59) & - & -.97 & .35 \\
\hline
\end{tabular}

encontrando-se agora num mesmo fator, revelando fortes correlações entre os mesmos, e apresentando também comunalidades superiores a .65. Denominamos este novo fator "Eficácia do coping e Autoestima". A junção destes dois domínios num só poderá justificar-se pelo facto de apresentarem entre eles uma relação direta, ou seja, um coping mais eficaz está frequentemente associado a uma autoestima mais elevada (Joaquín-Mingorance et al., 2019). Assim, as doentes capazes de desenvolver estratégias de coping eficazes apresentarão muito provavelmente uma perceção do self mais positiva que se traduzirá em níveis de autoestima mais elevados.

$\mathrm{O}$ segundo componente evidenciou saturações elevadas em 6 itens (Q10 a Q15) (ver Quadro 2), sendo de realçar, no sentido menos positivo, a carga fatorial apresentada pelo item $11(\mathrm{Q} 11=.49)$ que se encontra um pouco abaixo do limite aceitável de .60, no entanto, devido ao facto de este item se encontrar correlacionado com os restantes, optou-se por não o eliminar (Damásio, 2012). Os itens deste componente 2 são os mesmos que Biesecker et al. (2013) encontraram para a subescala "Integração social", com exceção do item Q10 (Q10=.81), que no estudo original integrava a subescala da "Autoestima". A denominação do componente 2 prevaleceu a mesma, "Integração Social" dado que o conteúdo do item 10 (Q10: "Ajudou-me a tornar numa pessoa melhor.") poderá também ser associado ao contexto social. Finalmente, o terceiro componente apresentou saturações elevadas em 5 itens (Q16 a Q20), com cargas fatoriais em valor absoluto superiores a .62. Este componente é semelhante ao da subescala do "Bem-Estar Espiritual" encontrada no estudo de Biesecker et al. (2013), apresentando uma estrutura que revela fortes correlações entre os seus itens e o total da subescala ( $r \geq .67)$ assim como valores de comunalidade superiores a .70. (ver Quadro 2).

\section{Fiabilidade}

A fiabilidade da escala total bem como de cada subescala foi avaliada através da análise da consistência interna, através do cálculo do valor de alfa de Cronbach. A escala total evidenciou um nível de consistência interna elevado $(\alpha=.85)$ e as três subescalas também apresentaram valores de consistência interna elevados, variando o valor de alfa de Cronbach entre $\alpha=.92$, para as subescalas "Integração social" e "Bem-Estar Espiritual" e $\alpha=.95$ para a subescala da "Eficácia do coping e Autoestima” (ver Quadro 1).

\section{Validade Externa}

Foram realizadas várias análises de correlação com outras medidas já validadas relacionadas com 
Quadro 2. Psychological Adaptation Scale (PAS): Cargas fatoriais da análise fatorial exploratória com rotação Oblimin $(\mathrm{N}=98)$

\begin{tabular}{|c|c|c|c|c|c|}
\hline \multirow{2}{*}{ Itens } & \multicolumn{3}{|c|}{ Componentes } & \multirow{2}{*}{ Comunalidades } & \multirow{2}{*}{$\begin{array}{l}\text { Alfa de Cronbach se o } \\
\text { item for eliminado }\end{array}$} \\
\hline & 1 & 2 & 3 & & \\
\hline Q1 & .82 & .15 & .03 & .80 & .94 \\
\hline Q2 & .84 & .20 & .14 & .81 & .94 \\
\hline Q3 & .87 & -.004 & .14 & .65 & .95 \\
\hline Q4 & .95 & -.06 & .06 & .79 & .95 \\
\hline Q5 & .73 & -.12 & -.33 & .76 & .94 \\
\hline Q6 & .60 & .06 & -.27 & .67 & .95 \\
\hline Q7 & .60 & .06 & -.33 & .75 & .94 \\
\hline Q8 & .68 & .07 & -.20 & .72 & .94 \\
\hline Q9 & .65 & .10 & -.26 & .78 & .94 \\
\hline Q10 & .09 & .81 & -.04 & .78 & .90 \\
\hline Q11 & .12 & .49 & -.10 & .39 & .93 \\
\hline Q12 & .04 & .88 & .06 & .77 & .90 \\
\hline Q13 & .10 & .71 & -.16 & .76 & .90 \\
\hline Q14 & -.11 & 1.02 & .07 & .85 & .90 \\
\hline Q15 & -.03 & .84 & -.13 & .81 & .90 \\
\hline Q16 & .06 & .16 & -.74 & .75 & .90 \\
\hline Q17 & .17 & .23 & -.62 & .79 & .89 \\
\hline Q18 & .21 & .18 & -.66 & .83 & .88 \\
\hline Q19 & .19 & .06 & -.72 & .77 & .91 \\
\hline Q20 & -.12 & .02 & -.89 & .71 & .93 \\
\hline
\end{tabular}

Quadro 3. Medidas descritivas (mínimo e máximo, média e desvio-padrão): DASS, FACT-G, FACIT-Sp e WHOQOL-Bref

\begin{tabular}{lcc}
\hline Instrumentos & Min-Máx & M (DP) \\
\hline DASS (Ansiedade, Depressão e Stress) & & $4.95(4.60)$ \\
Ansiedade (0-21) & $0-20$ & $4.61(4.84)$ \\
Depressão (0-21) & $0-19$ & $7.62(4.88)$ \\
Stress (0-21) & $0-21$ & \\
FACT-G (Bem-Estar) & $0-28$ & $19.64(6.06)$ \\
Bem-estar Físico (0-28) & $10-28$ & $22.89(4.42)$ \\
Bem-estar Social (0-28) & $3-24$ & $17.15(4.43)$ \\
Bem-estar Emocional (0-24) & $5-28$ & $19.17(5.47)$ \\
Bem-estar Funcional (0-28) & $32-108$ & $78.85(16.11)$ \\
Total (0-108) & & \\
FACIT-Sp (Bem-Estar Espiritual) & $0-32$ & $24.20(6.58)$ \\
Sentido/Paz (0-32) & $0-16$ & $11.16(4.18)$ \\
Fé (0-16) & $0-48$ & $35.36(9.37)$ \\
Total (0-48) & & \\
WHOQOL-Bref (Qualidade de Vida) & $0-100$ & $60.20(20.80)$ \\
Domínio Geral (0-100) & $11-100$ & $65.05(19.25)$ \\
Domínio 1 (0-100) & $4-100$ & $70.58(20.23)$ \\
Domínio 2 (0-100) & $0-100$ & $71.26(20.63)$ \\
Domínio 3 (0-100) & $25-100$ & $69.55(16.26)$ \\
Domínio 4 (0-100)
\end{tabular}

a AP (Biesecker et al., 2013; Cotton et al., 1999), com o intuito de analisar a validade externa do instrumento em estudo, supondo que a mesma apresentava uma correlação direta e estatisticamente significativa com os constructos de QdV, BE e BEE, e inversa com os constructos de Ansiedade, Depressão e Stress (Maroco, 2010).
Assim, torna-se pertinente conhecer a caracterização da amostra em termos de QdV, BE, BEE Ansiedade, Depressão e Stress, constructos avaliados através dos seguintes instrumentos: WHOQOL-Bref, FACT-G, FACIT-Sp, e DASS.

A nossa amostra apresenta níveis de QdV, $\mathrm{BE}, \mathrm{BEE}$ acima do ponto médio das respetivas 
Quadro 4. Validade externa: Correlações entre a PAS e os instrumentos: WHOQOL-Bref; FACT-G; FACIT$\mathrm{Sp}$; DASS $(\mathrm{N}=95)$

\begin{tabular}{|c|c|c|c|c|c|}
\hline & \multirow{2}{*}{ Instrumentos } & \multicolumn{4}{|c|}{ PAS } \\
\hline & & Subescala 1 & Subescala 2 & Subescala 3 & Total \\
\hline \multirow{10}{*}{ 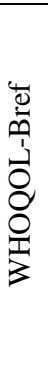 } & \multirow{2}{*}{ Domínio Físico } & $r=.31 * *$ & $r=.05$ & $r=.22 *$ & $r=.23^{*}$ \\
\hline & & $p=.002$ & $p=.61$ & $p=.03$ & $p=.02$ \\
\hline & \multirow{2}{*}{ Domínio Psicológico } & $r=.37 * *$ & $r=.14$ & $r=.34 * *$ & $r=.32 * *$ \\
\hline & & $p<.001$ & $p=.18$ & $p=.001$ & $p=.001$ \\
\hline & Domínio das Relações & $r=.23 *$ & $r=.16$ & $r=.20$ & $r=.22 *$ \\
\hline & Sociais & $p=.02$ & $p=.12$ & $p=.06$ & $p=.03$ \\
\hline & \multirow{2}{*}{ Domínio do Ambiente } & $r=.29 * *$ & $r=.14$ & $r=.23 *$ & $r=.26^{*}$ \\
\hline & & $p=.004$ & $p=.16$ & $p=.02$ & $p=.01$ \\
\hline & \multirow{2}{*}{$\begin{array}{l}\text { Qualidade de Vida Geral } \\
\text { (DG) }\end{array}$} & $r=.45 * *$ & $r=.37 * *$ & $r=.42 * *$ & $r=.46 * *$ \\
\hline & & $p<.001$ & $p<.001$ & $p<.001$ & $p<.001$ \\
\hline \multirow{10}{*}{ 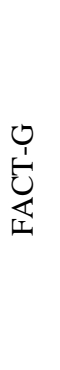 } & \multirow{2}{*}{ Bem-Estar Físico } & $r=.21 *$ & $r=.01$ & $r=.11$ & $r=.14$ \\
\hline & & $p=.04$ & $p=.92$ & $p=.28$ & $p=.17$ \\
\hline & \multirow{2}{*}{ Bem-Estar Social } & $r=.29 * *$ & $r=.29 * *$ & $r=.30 * *$ & $r=.33 * *$ \\
\hline & & $p=.003$ & $p=.004$ & $p=.003$ & $p=.001$ \\
\hline & \multirow{2}{*}{ Bem-Estar Emocional } & $r=.50 * *$ & $r=.26^{*}$ & $r=.37 * *$ & $r=.43 * *$ \\
\hline & & $p<.001$ & $p=.01$ & $p<.001$ & $p<.001$ \\
\hline & \multirow{2}{*}{ Bem-Estar Funcional } & $r=.42 * *$ & $r=.13$ & $r=.29 * *$ & $r=.33 * *$ \\
\hline & & $p<.001$ & $p=.19$ & $p=.004$ & $p=.001$ \\
\hline & \multirow{2}{*}{ Bem-Estar Total } & $r=.44 * *$ & $r=.20 *$ & $r=.32 * *$ & $r=.37 * *$ \\
\hline & & $p<.001$ & $p=.05$ & $p=.001$ & $p<.001$ \\
\hline \multirow{6}{*}{ 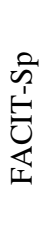 } & \multirow{2}{*}{ Sentido/Paz } & $r=.42 * *$ & $r=.17$ & $r=.39 * *$ & $r=.38 * *$ \\
\hline & & $p<.001$ & $p=.09$ & $p<.001$ & $p<.001$ \\
\hline & \multirow{2}{*}{ Fé } & $r=.36 * *$ & $r=.16$ & $r=.53 * *$ & $r=.39 * *$ \\
\hline & & $p<.001$ & $p=.12$ & $p<.001$ & $p<.001$ \\
\hline & \multirow[t]{2}{*}{ Total } & $r=.46 * *$ & $r=.19$ & $r=.51 * *$ & $r=.44 * *$ \\
\hline & & $p<.001$ & $p=.06$ & $p<.001$ & $p<.001$ \\
\hline \multirow{8}{*}{ 恣 } & \multirow{2}{*}{ Ansiedade } & $r=-.27 * *$ & $r=-.12$ & $r=-.19$ & $r=-.23^{*}$ \\
\hline & & $p=.008$ & $p=.25$ & $p=.07$ & $p=.003$ \\
\hline & \multirow{2}{*}{ Depressão } & $r=-.50 * *$ & $r=-.23 *$ & $r=-.45 * *$ & $r=-.45 * *$ \\
\hline & & $p<.001$ & $p=.02$ & $p<.001$ & $p<.001$ \\
\hline & \multirow{2}{*}{ Stress } & $r=-.38 * *$ & $r=-.21 *$ & $r=-.36 * *$ & $r=-.36 * *$ \\
\hline & & $p<.001$ & $p=.04$ & $p<.001$ & $p<.001$ \\
\hline & \multirow{2}{*}{ Total } & $r=-.42 * *$ & $r=-.21 *$ & $r=-.37 * *$ & $r=-.38 * *$ \\
\hline & & $p<.001$ & $p=.04$ & $p<.001$ & $p<.001$ \\
\hline
\end{tabular}

**A correlação é significativa no nível de 0.01. * A correlação é significativa no nível de .05 .

Legenda: Subescala 1: "Eficácia do Coping e Autoestima"; Subescala 2: "Integração Social”; Subescala 3: "Bem-Estar Espiritual”;

$r=$ Coeficiente de Pearson; $p=$ nível de significância.

Nota. Os resultados identificados a negrito correspondem às correlações estatisticamente significativas.

escalas. Quanto aos níveis de Ansiedade, Depressão e Stress, verificou-se que apresenta resultados inferiores ao ponto médio das escalas, $o$ que revela níveis pouco elevados de Ansiedade, Depressão e Stress. Uma possível explicação para esses valores poderá ter a ver com características específicas da amostra em causa como é o caso do tempo decorrido após o diagnóstico. DukesHolland e Holahan (2003) referem que em média é necessário um período de cerca de um ano para a adaptação dos doentes oncológicos à doença. Ora a média de tempo após o diagnóstico da amostra deste estudo é de cerca de 3 anos, podendo este tempo pós diagnóstico ser já o suficiente para normalizar os níveis de Ansiedade, Depressão e Stress e funcionar também como um adjuvante na promoção da QdV, BE e BEE. Quadro 3).

Passando agora para a análise das correlações obtidas entre os constructo da AP e constructos anteriormente referidos, começamos por analisar a correlação entre os resultados da PAS (AP) e os resultados do WHOQOL-Bref (QdV) (ver Quadro 4), realçando os valores positivos e estatisticamente significativos do resultado global da PAS com todos os domínios da QdV incluindo o Domínio Geral da QdV, apresentando este último o valor mais elevado $(r=.46 ; p<.001)$. Quanto às três subescalas da PAS, verificou-se que a subescala "Eficácia do Coping e Autoestima" apresentou correlações positivas e significativas com todos os domínios da QdV. 
Estes resultados corroboram os resultados de outros estudos em que se verificam associações significativas entre a eficácia do coping e a autoestima na QdV (Santos \& Ribeiro, 2001; Sendim, 2017). Relativamente à subescala "BemEstar Espiritual", verificaram-se correlações positivas e estatisticamente significativa com quase todos os domínios da $\mathrm{QdV}$, à exceção do Domínio das Relações Sociais. Finalmente, a subescala "Integração Social" da PAS, à exceção do Domínio Geral de QdV, não apresentou correlações significativas com nenhum dos outros domínios do WHOQOL-Bref, nem mesmo com o Domínio das Relações Sociais. Este resultado poderá ser atribuído ao facto da PAS e do WHOQOL-Bref serem instrumentos que avaliam a dimensão das relações sociais de forma diferente, uma vez que o primeiro instrumento procura avaliar as relações sociais enquanto processo, ao passo que o WHOQOL-Bref o avalia como resultado final através da satisfação que os doentes revelam com os seus contextos socias.

Passando à análise das correlações dos resultados da PAS com os resultados do FACT-G que visa avaliar a QdV relacionada com a Saúde especificada em 4 domínios de BE (ver Quadro 4), verificamos um padrão de correlações semelhantes às encontradas com o WHOQOLBref. Assim, verificamos que tanto a PAS total como os da subescala "Eficácia do Coping e Autoestima" da PAS apresentaram correlações positivas e significativas com todos os domínios do FACT-G à exceção do domínio do Bem-Estar Físico. A subescala "Bem-Estar Espiritual" também se correlaciona positiva e significativamente com todos os domínios do FACT-G menos um, tal como aconteceu com a WHOQOL-Bref, só que neste caso essa exceção é o domínio do Bem-Estar Físico. Por último, a subescala "Integração Social" é a que menos se relaciona com o FACT-G, tal como já tinha acontecido para a WHOQOL-Bref. Apresentou correlações positivas e estatisticamente significativas, apesar de os valores serem abaixo de 0.30, com o Bem-Estar Social $(r=.29, p=.004)$, o Bem-Estar Emocional ( $r=.26, p=.01)$ e o BemEstar Total $(r=.20, p=.05)$, e não apresentou correlações estatisticamente significativas nem com o Bem-Estar Físico nem com o Bem-estar Funcional. Podendo ser um indicador de que a
PAS e as suas subescalas se focam pouco no domínio físico. Estes resultados, de uma maneia geral, apontam, de acordo com o esperado, para a existência de uma relação positiva entre o constructo da AP e o constructo do BE.

Quanto às correlações entre a PAS e o BEE mensurado pelo FACIT-Sp verificou-se que os valores, tal como esperado, se revelaram estatisticamente significativos para a subescala do "Bem-Estar Espiritual" (ver Quadro 4).

Observaram-se assim, no sentido esperado, correlações estatisticamente significativas entre a AP e os constructos QdV, BE e BEE. De salientar que os valores relativos a essas correlações são em geral moderados confirmando a ideia de que apesar de os constructos estarem relacionados, não avaliam exatamente $\mathrm{o}$ mesmo, não se sobrepondo, e evidenciando assim a pertinência de um instrumento dirigido especificamente para a $\mathrm{AP}$, que integra dimensões não abrangidas pelos constructos da QdV, do BE e do BEE (Biesecker et al., 2013).

Por fim, com o intuito de analisar a relação da PAS com outros constructos, mas agora em sentido inverso, recorreu-se aos resultados obtidos na DASS que avalia a Ansiedade, Depressão e o Stress, esperando obter valores de correlações inversas e estatisticamente significativas (Biesecker et al., 2013) (ver Quadro 4).Observou-se que tanto a PAS Total como a subescala "Eficácia do Coping e Autoestima" se correlacionaram de forma inversa e estatisticamente significativa com todos os domínios da DASS. Quanto às subescalas "Integração Social" e "Bem-Estar Espiritual", verificaram-se correlações negativas e estatisticamente significativas com a DASS Total e dois dos seus domínios: Depressão e do Stress. Assim, e de acordo com o esperado verificou-se que na sua maioria estes constructos se correlacionam de forma negativa e significativa com a PAS.

Os resultados das correlações realizadas permitem-nos considerar que a PAS é um instrumento com validade externa dado que as correlações obtidas foram no sentido esperado, ou seja, apresentando correlações positivas e significativas com os constructos QdV, BE e BEE e correlações negativas e estatisticamente significativas com os constructos Ansiedade, Depressão e Stress. 


\section{Análise descritiva da PAS}

Fazendo uma análise descritiva da PAS e das suas subescalas (ver Quadro 1), constata-se que a subescala da "Eficácia do coping e Autoestima" apresenta uma média de $31.95 \quad(D P=8.79$; amplitude possível: $\min .=9$ máx. $=45$ ) valor bastante acima do ponto médio da subescala - 27 , e indicando que as pacientes desta amostra percecionam dispor de estratégias de coping eficazes e de valores elevados de autoestima, estes resultados são corroborados por vários estudos que referem que no caso do cancro da mama há muitas doentes oncológicas que conseguem desenvolver estratégias eficazes de enfrentamento da doença (Brandão et al., 2017; Moorey \& Greer, 2002; Moreira \& Canavarro, 2011). Quanto à subescala da "Integração Social" a média dos resultados $21.74(D P=6.16$; amplitude possível: min.=5 máx.=30) encontra-se também acima do ponto médio da subescala -18 , mostrando que estas mulheres se parecem sentir integradas nos respetivos meios sociais, aspeto referido por vários autores como um fator protetor quanto ao processo de ajustamento à doença oncológica (Ávila et al., 2015; Kang et al., 2020). Relativamente à subescala do "Bem-Estar Espiritual" foram observados valores médios de 17.98 ( $D P=5.54$; amplitude possível: $\min .=5$ máx. $=25$ ), próximos do ponto médio da subescala -17.5 parecendo evidenciar que estas mulheres apresentam um certo bem-estar espiritual, tendo vários autores referido a importância da espiritualidade nos processos adaptativos positivos (Fitchett et al., 1996; Kang et al., 2020; Ponte \& Pais-Ribeiro, 2016; Visser et al., 2010).

Para finalizar a análise descritiva da PAS, esta amostra apresentou um valor médio de 71.48 $(D P=18.59$; amplitude possível: $\min .=20$ máx.=100), situando-se este valor acima do ponto médio da escala total - 70, indicando que em geral esta amostra revela uma boa adaptação psicológica à doença.

Realizaram-se também análises com vista a analisar a relação da PAS com algumas características sociodemográficas (idade, número de filhos, número de anos de escolaridade, estado civil e situação profissional) e clínicas (o tempo desde o diagnóstico, acompanhamento psicológico aquando do processo da doença, estádio da doença e apresentação de metástases), tendo-se apenas revelado como estatisticamente significativas as diferenças relativas ao estado civil e ao acompanhamento psicológico.

Mulheres com companheiros/as $(M=75.64$; $D P=14.54)$ revelaram níveis mais elevados de AP por comparação com mulheres sem companheiros/as $(M=55.25 ; \quad D P=23.63)$, sendo esse resultado verificado quer para a escala total da PAS $[t(22.81)=-3.68 ; p=.001]$ quer para as suas 3 subescalas, nomeadamente, na subescala da "Eficácia do Coping e Autoestima" [t(23.67)=3.84; $p=.001$ ], na subescala da "Integração Social" $[t(23.77)=-3.06 ; p=.005]$ e na subescala do "BemEstar Espiritual" $[t(22.81)=-3.00 ; p=.006]$. Este resultado vem realçar a importância do suporte emocional dos companheiros/as no processo de enfrentamento da doença, aspeto também referido no estudo de Ávila et al. (2015).

O acompanhamento psicológico revelou ser benéfico em termos de AP, tendo as mulheres, que receberam apoio psicológico no decurso da doença, apresentado valores de "Integração Social" superiores $(M=23.91 ; D P=4.34)$ aos das mulheres que não receberam qualquer tipo de apoio $(M=21.12 ; D P=6.48)$, salientando assim o efeito positivo desse tipo de intervenção terapêutica $[t(50.88)=5.53 ; p=.023]$.

\section{Conclusão}

A doença oncológica é uma doença exigente não só física como emocionalmente, levando frequentemente a estados de ansiedade, depressão e stress, traduzindo-se frequentemente em processos de adaptação à doença mais complexos.

Para contrariar a espiral negativa, em que as doentes facilmente entram quando confrontadas com um diagnóstico de cancro da mama, é fundamental a identificação precoce de padrões de funcionamento emocional menos funcionais, de forma a poder promover o desenvolvimento de estratégias de coping eficazes como o espírito de luta e/ou da aceitação, o fortalecimento da sua autoestima e a integração social destas mulheres, encaminhando-as assim para processos de adaptação à doença oncológica mais positivos, que simultaneamente restabeleçam a sua $\mathrm{QdV}$ e o seu BE.

É neste sentido que surge a pertinência desta investigação ao apresentar uma proposta de 
validação da versão portuguesa de um instrumento - PAS - que visa avaliar as várias dimensões que compõem a AP.

Através de uma análise fatorial exploratória, foi obtida uma solução fatorial que explica $74.65 \%$ da variância, que se estrutura em 3 subescalas: "Eficácia do coping e Autoestima" com 9 itens; "Integração Social" com 6 itens; e "Bem-Estar Espiritual" com 5 itens, e que divergiu da solução original encontrada por Biesecker et al. (2013) bem como da solução fatorial encontrada por Jönsson (2018).

Em termos de validade externa, a PAS mostrou ser um instrumento válido, que apresenta uma correlação positiva e estatisticamente significativa com outros constructos relacionados com a AP, nomeadamente a $\mathrm{QdV}$, o BE e o BEE. Também evidenciou correlações negativas e estatisticamente significativas com construtos opostos à AP, nomeadamente, Ansiedade, Depressão e Stress.

Relativamente à fiabilidade, avaliada por intermédio do cálculo do alfa de Cronbach, obtiveram-se valores superiores a .85 tanto para a escala total como para as suas subescalas, levandonos a concluir que se trata de um instrumento fiável.

A PAS parece assim ser um instrumento dotado de boas qualidades psicométricas, sendo válido e fiável nesta amostra de doentes oncológicas com cancro da mama.

Existem neste estudo, todavia, algumas limitações que não se poderá deixar de referir, começando pelas características da amostra, que apresenta apenas um tipo de neoplasia e contempla apenas o género feminino, dificultando assim generalizações para a população oncológica. A extensão do protocolo de questionários é também um aspeto menos positivo, uma vez que o torna bastante moroso. O momento de abordagem das participantes para participarem no estudo pode também ter constituído um ponto menos positivo, uma vez que foi realizado após as consultas e/ou tratamentos com o intuito de não interferir nos mesmos, o que, contudo, levou a que algumas participantes se encontrassem cansadas para o preenchimento dos questionários. O caráter transversal deste estudo constitui ainda uma última limitação não permitindo a realização de algumas análises como é o caso do teste-reteste para a análise da fiabilidade.
Numa futura investigação sugere-se a realização de um estudo longitudinal com o propósito de avaliar a AP em momentos diferentes do processo de doença.

Termina-se salientando a pertinência deste estudo ao mostrar a validade da versão portuguesa da PAS, um instrumento que caracteriza e descreve de forma abrangente o processo de AP, composto por diferentes dimensões, e que se distingue de outras medidas tradicionalmente relacionadas com a AP como é o caso da $\mathrm{QdV}$ e do BE. Apesar de este instrumento já ter contado com uma primeira validação da versão em língua portuguesa proposta por Jönsson em 2018, esta não visava especificamente pessoas com doença, mas sim pessoas com acontecimentos de vida stressantes, não especificamente relacionados com a saúde. O presente estudo, vem assim assegurar a viabilidade da utilização da PAS no contexto da doença oncológica. Ao possibilitar a identificação de eventuais domínios de AP mais problemáticos, poderá revelar-se um instrumento muito útil em especial na estruturação de programas de acompanhamento dirigidos para o desenvolvimento das componentes da AP menos funcionais e para a intervenção psicológica em geral.

\section{Referências}

Alberts, D., Lluria-Prevatt, M., Kha, S., \& Weihs, K. (Eds.). (2016). Supportive cancer care. Arizona: Springer.

Antoni, M., Lechner, S., Diaz, A., Vargas, S., Holley, H., Phillips, K., McGregor, B., Carver, C., \& Blomberg, B., (2009). Cognitive behavioral stress management effects on psychosocial and physiological adaptation in women undergoing treatment for breast cancer. Brain, behavior, and immunity, 23(5), 580-591.

Ávila, M., Brandão, T., Teixeira, J., Coimbra, J., \& Matos, P. (2015). Attachment, emotion regulation, and adaptation to breast cancer: Assessment of a mediational hypothesis. Psycho-Oncology, 24(11), 1514-1520.

Biesecker, B., Erby, L., Woolford, S., Adcock, J., Cohen, J., Lamb, A., Lewis, K., Truitt, M., Turriff, A., \& Reeve, B., (2013). 
Development and validation of the Psychological Adaptation Scale (PAS): Use in six studies of adaptation to a health condition or risk. Patient Education and Counseling, 93(2), 248-254.

Borsa, J., Damásio, B., \& Bandeira, D. (2012). Adaptação e validação de instrumentos psicológicos entre culturas: Algumas considerações. Paidéia

(Ribeirão Preto), 22(53), 423-432.

Brandão, T., \& Matos, P. (2015). Eficácia das intervenções psicológicas em grupo dirigidas a mulheres com cancro da mama: Uma revisão sistemática. Revista Portuguesa de Saúde Pública, 33(1), 98-106.

Brandão, T., Schulz, M., \& Matos, P. (2017). Psychological adjustment after breast cancer: A systematic review of longitudinal studies. Psycho-oncology, 26(7), 917-926.

Brazão, I. (2020). A psicoeducação no controle da ansiedade na mulher com cancro da mama: Proposta de intervenções especializadas em enfermagem (Dissertação de Mestrado, Instituto Politécnico de Portalegre).

Cardoso, G., Luengo, A., Trancas, B., Vieira, C., \& Reis, D. (2009). Aspectos psicológicos do doente oncológico. Psicólogos: Revista do Serviço de Psiquiatria do Hospital Prof. Dr. Fernando Fonseca, EPE, 8-18.

Cotton, S., Levine, E., Fitzpatrick, C., Dold, K., \& Targ, E. (1999). Exploring the relationships among spiritual well-being, quality of life, and psychological adjustment in women with breast cancer. Psycho-Oncology: Journal of the Psychological, Social and Behavioral Dimensions of Cancer, 8(5), 429-438.

Cella, D., Tulsky, D., Gray, G., Sarafian, B., Linn, E., Bonomi, A., Silberman, M., Yellen, S., Winicour, P., Brannon, J., Eckberg, K., Lloyd, S., Prul, S., Blendowski, C., Goodman, M., Barnicle, M., Stewart, I., McHale, M., Bonomi, P., Kaplan, E., Taylor IV, S., Thomas Jr, C., \& Harris, J. (1993). The Functional Assessment of Cancer Therapy scale: development and validation of the general measure. Journal of Clinical Oncology, 11(3), 570-579.

Damásio, B. (2012). Uso da análise fatorial exploratória em psicologia. Avaliação
Psicologica: Interamerican Journal of Psychological Assessment, 11(2), 213-228.

Dukes-Holland, K., \& Holahan, C. (2003). The relation of social support and coping to positive adaptation to breast cancer. Psychology and Health, 18(1), 15-29.

Dumalaon-Canaria, J., Prichard, I., Hutchinson, A. D., \& Wilson, C. (2018). Fear of cancer recurrence and psychological well-being in women with breast cancer: The role of causal cancer attributions and optimism. European Journal of Cancer Care, 27(1). doi:10.1111/ecc.12579

Espírito Santo, H., \& Daniel, F. (2017). Calcular e apresentar tamanhos do efeito EM trabalhos científicos (1): As limitações do $\mathrm{P}<0.05$ na análise de diferenças de médias de dois grupos (calculating and reporting effect sizes on scientific papers (1): $\mathrm{P}<0.05$ Limitations in the analysis of mean differences of two groups). Revista Portuguesa de Investigação Comportamental e Social, 1(1), 3-16.

Field, A. (2013). Discovering statistics using IBM SPSS Statistics $\left(4^{\text {a }}\right.$ ed.). London: SAGE Publications Ltd.

Fitchett, G., Peterman, A., \& Cella, D. (1996) Spiritual beliefs and quality of life in cancer patients and HIV patients. Paper presented at The Third World Congress of PsychoOncology, October 3-6, New York, NY.

Friedman, L., Nelson, D., Baer, P., Lane, M., \& Smith, F. (1991). Adjustment to breast cancer: A replication study. Journal of Psychosocial Oncology, 8(4), 27-40.

Gudmundsson, E. (2009). Guidelines for translating and adapting psychological instruments. Nordic Psychology, 61, 29-45.

Heim, E., Valach, L., \& Schaffner, L. (1997). Coping and psychosocial adaptation: Longitudinal effects over time and stages in breast cancer. Psychosomatic Medicine, 59(4), 408-418.

Holland, J. (Ed.3). (2010). Psycho-oncology. New York, NY: Oxford University Press.

IBM SPSS (2017). IBM SPSS Statistics Base 25. Chicago, IL: SPSS Inc.

Joaquín-Mingorance, M., Arbinaga, F., CarmonaMárquez, J., \& Bayo-Calero, J. (2019). Coping strategies and self-esteem in women with breast cancer. Anales de 
Psicología/Annals of Psychology, 35(2), 188194.

Jonsson, C. (2018). Theodicies and Psychological Adaptation. (Tese de Doutoramento, Universidade do Porto).

Kang, N., Kim, H., Kim, J., \& Kim, S. (2020). Relationship between cancer stigma, social support, coping strategies and psychosocial adjustment among breast cancer survivors. Journal of Clinical Nursing, 29(2122), 4368-4378.

Lang-Rollin, I., \& Berberich, G. (2018). Psychooncology. Dialogues in clinical neuroscience, 20(1), 13.

Larsen, P. (2014). Psychosocial Adjustment in Lubkin's Chronic Illness. Wyoming, USA: Jones \& Bartlett Publishers.

Lazarus, R., \& Folkman, S. (1984). Stress, appraisal and coping. New York: Springer.

Loeffler, S., Poehlmann, K., \& Hornemann, B. (2018). Finding meaning in suffering?Meaning making and psychological adjustment over the course of a breast cancer disease. European Journal of Cancer Care, 27(3). doi:10.1111/ecc.12841.

Maroco, J. (2010). Análise de equações estruturais: Fundamentos teóricos, softwares $e$ aplicações. Pêro Pinheiro: Produtos e Serviços de Estatística, Lda.

Moorey, S., \& Greer, S. (2002). Cognitive behaviour therapy for people with cancer. Oxford: Oxford University Press.

Moreira, H., \& Canavarro, M. C. (2011). A adaptação ao cancro da mama nas fases de diagnóstico e sobrevivência: Será o investimento na aparência um factor explicativo relevante? Análise Psicológica, 29(4), 505-520.

Moreira, H., Silva, S., \& Canavarro, C. (2008). Qualidade de vida e ajustamento psicossocial da mulher com cancro da mama: Do diagnóstico à sobrevivência. Psicologia, Saúde \& Doenças, 9(1), 165-184.

National Comprehensive Cancer Network (NCCN). (2021). Patient and Caregiver Resources: Managing Stress and Distress Disponível em: https://www.nccn.org/patients/resources/life_ with_cancer/distress.aspx. Acedido em: Março de 2021.
Pais-Ribeiro, J., Honrado, A., \& Leal, I. (2004). Contribuição para o estudo da adaptação portuguesa das escalas de ansiedade, depressão e stress (EADS) de 21 itens de Lovibond e Lovibond. Psicologia, Saúde \& Doenças, 5(2), 229-239.

Pereira, F., \& Santos, C. (2011). Adaptação cultural da Functional Assessment of Chronic ILLness Therapy-Spiritual Well-Being (FACIT-Sp): Estudo de validação em doentes oncológicos na fase final de vida. Cadernos de Saúde, 4(2), 37-45.

Pinto, C., \& Pais-Ribeiro, J. (2007). Sobrevivente de cancro: Uma outra realidade. Texto Contexto Enferm, 16(1), 142-8.

Ponte, A., \& Pais-Ribeiro, J. (2016). O bem-estar do doente paliativo: Revisão bibliográfica. Cadernos de Saúde, 8, 38-55.

Riba, M., Donovan, K., Andersen, B., Braun, I., Breitbart, W., Brewer, B., Buchmann, L., Clark, M., Collins, M., Corbett, C., Fleishman, S., Garcia, S., Greenber, D., Handzo, G., Hoofrinh, L., Huang, C., Lally, R., Martin, S., McGuffey, L., ... \& Darlow, S. (2019). Distress management, version 3.2019, NCCN clinical practice guidelines in oncology. Journal of the National Comprehensive Cancer Network, 17(10), 1229-1249.

Santos, C., \& Ribeiro, J. (2001). A qualidade de vida do doente oncológico: Revisão bibliográfica. Referência, 7, 5-16.

Sendim, S. (2017). Funcionalidade do membro superior, qualidade de vida e estratégias de coping da mulher submetida a cirurgia por cancro da mama (Dissertação de mestrado, Instituto Politécnico de Bragança).

Silva, S., Moreira, H., Pinto, S., \& Canavarro, M. (2009). Cancro da mama e desenvolvimento pessoal e relacional: Estudo das características psicométricas do Inventário de Desenvolvimento Pós-Traumático (Posttraumatic Growth Inventory) numa amostra de mulheres da população Portuguesa. Revista Iberoamericana de Diagnóstico y Evaluación - e Avaliação Psicológica, 2(28), 105-133.

Souza, J. (2014) Indicador de Risco Psicológico em Oncologia (IRPO): Construção e validação de um instrumento de triagem para 
pacientes com câncer. (Tese de Doutoramento, Universidade de Brasília).

Tanzer, N., \& Sim, Q. (1999) Adapting instruments for use in multiple languages and cultures: A review of the ITC guidelines for test adaptations. European Journal of Psychological Assessment, 3, 258-269.

Taylor, S. (1983). Adjustment to threatening events: A theory of cognitive adaptation. American psychologist,38(11), 1161.

Torres, A., Pereira, A., \& Monteiro, S. (2014). Estudo de validade da versão portuguesa do Questionário de Formas de Lidar com o Cancro. Revista Iberoamericana de Diagnóstico y Evaluación - e Avaliação Psicológica, 2(38), 199-220.

Vaz Serra, A., Canavarro, M., Simões, M., Pereira, M., Gameiro, S., Quartilho, M., Rijo, D., Carona, C., \& Paredes, T. (2006). Estudos psicométricos do instrumento de avaliação da qualidade de vida da Organização Mundial de Saúde (WHOQOL-Bref) para Português de Portugal. Psiquiatria clínica (Vol. 27, No. 1, pp. 41-49).

Visser, A., Garssen, B., \& Vingerhoets, A. (2010). Spirituality and well-being in cancer patients: a review. Psycho-Oncology: Journal of the Psychological, Social and Behavioral Dimensions of Cancer, 19(6), 565-572. 


\section{Anexo 1}

Itens da versão original de Biesecker et al. (2013): Psychological Adaptation Scale (PAS)

\begin{tabular}{ll}
\hline $\mathrm{N}^{\circ}$ item & Item \\
\hline Q1 & Helped me accept the way things work out. \\
Q2 & Helped me learn to deal better with uncertainty. \\
Q3 & Taught me how to adjust to things I cannot change. \\
Q4 & Helped me take things as they come. \\
Q5 & Helped me to look at things in a more positive way. \\
Q6 & Helped me learn to handle difficult times. \\
Q7 & Helped me become more comfortable with who I am. \\
Q8 & Helped me become a stronger person. \\
Q9 & Helped me feel better about my ability to handle problems. \\
Q10 & Helped me become a better person. \\
Q11 & Helped me know who I can count on in times of trouble. \\
Q12 & Makes me more willing to help others. \\
Q13 & Helped relationships become more meaningful. \\
Q14 & Helped me become closer to people I care about. \\
Q15 & Helped me become more aware of the love and support available from other people. \\
Q16 & Helped me learn my life is more meaningful. \\
Q17 & Given me a greater appreciation for life. \\
Q18 & Helped me develop a deeper sense of purpose in life. \\
Q19 & Helped me feel peaceful. \\
Q20 & Helped me find strength in my faith or Spiritual beliefs. \\
\hline
\end{tabular}

\section{Anexo 2}

Itens da versão portuguesa de Jönsson (2018): Escala de Adaptação Psicológica (EAP)

\begin{tabular}{ll}
\hline $\mathrm{N}^{\circ}$ item & Item \\
\hline Q1 & Ajudou-me a aceitar a forma como as coisas se resolvem. \\
Q2 & Ajudou-me a aprender a lidar melhor com a incerteza. \\
Q3 & Ajudou-me a ajustar às coisas que não posso mudar. \\
Q4 & Ajudou-me a lidar com as coisas à medida que vão surgindo. \\
Q5 & Ajudou-me a ver as coisas de uma forma mais positiva. \\
Q6 & Ajudou-me a aprender a lidar com tempos difíceis. \\
Q7 & Ajudou-me a sentir mais confortável com quem sou. \\
Q8 & Ajudou-me a tornar numa pessoa mais forte. \\
Q9 & Ajudou-me a sentir melhor quanto à minha capacidade para lidar com problemas. \\
Q10 & Ajudou-me a tornar numa pessoa melhor. \\
Q11 & Ajudou-me a saber com quem posso contar em tempos de dificuldades. \\
Q12 & Tornou-me mais disponível para ajudar os outros. \\
Q13 & Ajudou a que as relações com os outros tivessem mais significado. \\
Q14 & Ajudou-me a tornar mais próximo das pessoas com quem me preocupo. \\
Q15 & Ajudou-me a tornar mais consciente do amor e do apoio das outras pessoas. \\
Q16 & Ajudou-me a aprender que a minha vida tem mais significado. \\
Q17 & Deu-me uma maior estima pela vida. \\
Q18 & Ajudou-me a desenvolver um sentido mais profundo de propósito na vida. \\
Q19 & Ajudou-me a sentir paz. \\
Q20 & Ajudou-me a encontrar força na minha fé ou crenças espirituais. \\
\hline
\end{tabular}

